\title{
Evidence from a multicenter CMR registry indicates that stress CMR imaging provides highly effective risk stratification in patients suspected to have myocardial ischemia
}

\author{
Amit R Patel ${ }^{2}$, Kevin Steel ${ }^{4}$, Caroline A Daly ${ }^{5}$, Akhil Narang ${ }^{2}$, Subha V Raman ${ }^{3}$, Raymond Y Kwong ${ }^{1 *}$ \\ From 17th Annual SCMR Scientific Sessions \\ New Orleans, LA, USA. 16-19 January 2014
}

\section{Background}

A multi-center registry can provide robust real-world evidence regarding CMR diagnostic effectiveness and patient risk stratification, when imaging protocols, data collection, and reporting were standardized. Such evidence from the use of vasodilating stress CMR perfusion is currently limited.

\section{Methods}

In 2006, we developed a web-based multicenter registry (CMR-Cooperative, CMRCOOP) specific for performance of clinical CMR. This registry aimed to standardize imaging protocol, collection of clinical data, data interpretation, and reporting. All patient identifying information was encrypted. All data was stored and protected by intranet servers and site-specific administrative access. We identified patients who were referred for vasodilating CMR studies with suspected ischemia from 3 major CMR and 1 European centers. Presence of $>1$ segment of abnormal stress perfusion without LGE defines ischemia presence and the number of ischemic segments defines ischemia extent. Major hard outcomes (MACE) including all-cause mortality and acute MI were assessed and were associated with CMR evidence of ischemia, using Cox regression.

\section{Results}

From the 4 centers, 1729 patients were followed for MACE. At a median of 2.3 years, 146 patients $(8 \%)$ experienced MACE (80 deaths, 66 acute MIs). Ischemia

'Brigham and Women's Hospital, Boston, Massachusetts, USA

Full list of author information is available at the end of the article presence and ischemia extent both demonstrated strong association with MACE: Ischemia presence portended to a near 4-fold increase in MACE $(\mathrm{P}<0.0001)$; whereas for every segmental increase of ischemia extent, hazards of MACE increased by $14 \%(\mathrm{P}<0.0001)$. Kaplan-Meier survival curves are shown (Figure 1). Cumulative survival curves (Figure 2) demonstrated that vasodilating CMR appeared to have a high negative event rate for the initial 4 years after the CMR study.

\section{Conclusions}

From this multicenter web-based registry with standardized imaging and data-collection methods, vasodilating CMR demonstrates robust patient risk stratification in patients with suspected ischemia.

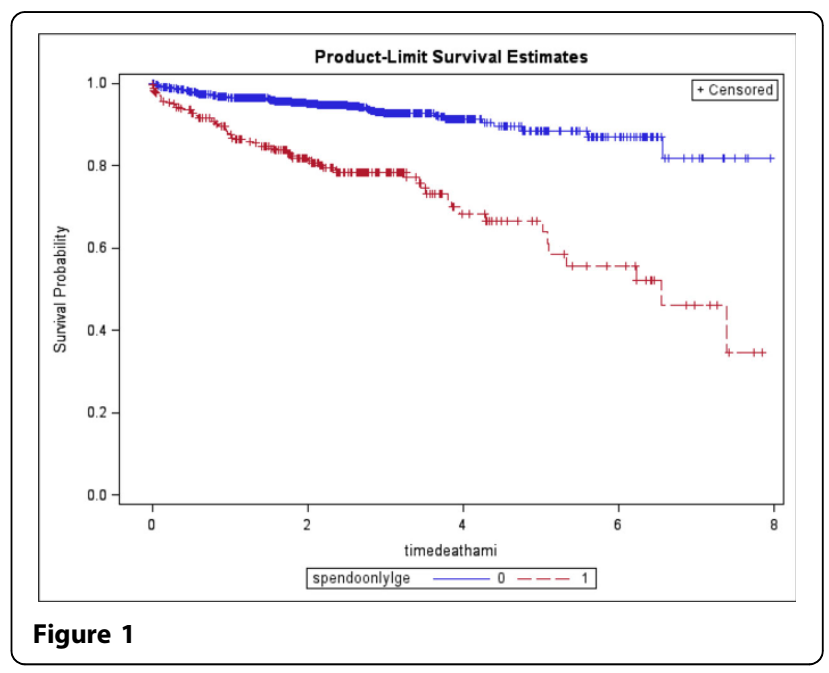




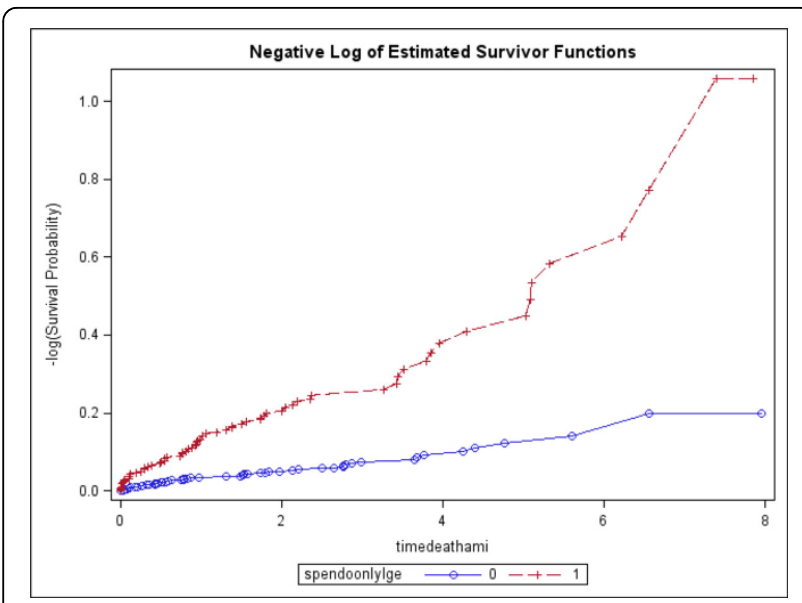

Figure 2

\section{Funding}

No external funding.

\section{Authors' details}

'Brigham and Women's Hospital, Boston, Massachusetts, USA. ${ }^{2}$ University of Chicago, Chicago, Illinois, USA. ${ }^{3}$ The Ohio State University Medical Center, Columbus, Ohio, USA. "Wilford Hall Medical Center, San Antonio, Texas, USA.

${ }^{5}$ St. James Hospital, Dublin, Ireland.

Published: 16 January 2014

doi:10.1186/1532-429X-16-S1-M1

Cite this article as: Patel et al:: Evidence from a multicenter CMR

registry indicates that stress CMR imaging provides highly effective risk

stratification in patients suspected to have myocardial ischemia. Journal of Cardiovascular Magnetic Resonance 2014 16(Suppl 1):M1.

Submit your next manuscript to BioMed Central and take full advantage of:

- Convenient online submission

- Thorough peer review

- No space constraints or color figure charges

- Immediate publication on acceptance

- Inclusion in PubMed, CAS, Scopus and Google Scholar

- Research which is freely available for redistribution

Submit your manuscript at www.biomedcentral.com/submit 